\title{
Array-Geometry Invariant Signaling for MISO Feedforward Opportunistic Communications
}

\author{
Jordi Borras and Gregori Vazquez \\ Department of Signal Theory and Communications, Technical University of Catalonia (UPC) \\ Email: \{jordi.borras.pino, gregori.vazquez\}@upc.edu
}

\begin{abstract}
The impact of antenna array geometry on scenarioaware invariant signaling is studied in the context of feedforward opportunistic communications. Invariance plays an elemental role to guarantee the detectability in noncooperative opportunistic systems. Specifically, we prove that received signal is independent of array geometry and hence time-domain invariance is preserved in a multiple-input single-output (MISO) communication with arbitrary array geometry. To address the latter, we exploit the idea of array manifold separation. Numerical simulations are reported to corroborate the theoretical contribution.
\end{abstract}

Index Terms-Opportunistic communications, array-geometry invariance, arbitrary array geometries, manifold separation.

\section{INTRODUCTION AND PRELIMINARIES}

Coordination is necessary in distributed communication systems to guarantee the desired system performance or, at least, make the communication itself feasible. For this reason, wireless terminals can design appropriate precoding schemes (see, e.g., [1], [2]) and perform the required interference management techniques (see, e.g., [3], [4]). Typically, coordination is achieved by means of hand-shake (feedback-based) schemes which exploit reference signals or pilots to agree some system parameters or acquire channel state information [5], [6].

Nevertheless, using reference signals incurs in a communication overhead that decreases the overall system performance by increasing latency and diminishing the information rate. As reported in [7], coordination outperforms noncooperative systems. However, cooperation cannot turn an interferencelimited regime to a noise-limited one [7].

The authors proposed in previous works the design of context- or scenario-aware invariant waveforms or signaling patterns. As reported in [8], [9], the use of this novel signaling patterns design scheme allows reducing the aforementioned communication overheads. The invariance property is the reason why hand-shake procedures can be simplified. As well, the scenario-adapted property provide these waveforms with interference management capabilities.

\section{A. Distributed Waveform Design}

The decentralized design of context-aware invariant waveforms was presented by the authors for the single-antenna case in [8], [10] as a minimum-norm problem, and generalized as a total least-squares in [9]. The latter can be immediately

The research leading to this work has been funded by the Spanish Ministry of Science and Innovation under the project WINTER: TEC2016-76409-C21-R (AEI/FEDER, UE) and fellowship FPI BES-2017-080071, and by the Catalan Government (AGAUR) through the grant 2017 SGR 578. extended to symmetric and structured multichannel scenarios [11]. For notation purposes, we briefly review this result.

Let us consider a heterogeneous wireless network composed of an unknown number of transmitting terminals. These terminals are exploiting several degrees of freedom (DoF) [12], [13]. A new transmitter-receiver pair wishes to opportunistically exploit the unused DoF. For this purpose, these new terminals have to sense which DoF are available and design an appropriate transmission-reception scheme. It is worth noting that the new terminals do not cooperate with other existing terminals and are completely uncoordinated. Under the lack of side information on existing transmissions and opportunistic node coordination, each new terminal has to define a random sensing basis which spans the whole ambient space and decide which DoF are available for opportunistic communication. Mathematically speaking, let $\boldsymbol{\Psi}(\mathrm{T}) \in \mathbb{C}^{N \times N}$ be a full-rank orthonormal random matrix. For the sake of illustration, index $\mathrm{T}$ denotes that $\boldsymbol{\Psi}(\mathrm{T})$ is the sensing basis at opportunistic transmitter. As reported in [8], [9], the design strategy at opportunistic receiver is symmetric. By leveraging this sensing basis, opportunistic transmitter can sense the network state

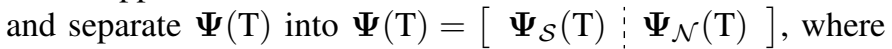
$\boldsymbol{\Psi}_{\mathcal{S}}(\mathrm{T})$ contains the occupied DoF and spans the so-called signal subspace, whereas the available DoF belong in the socalled noise subspace, spanned by $\Psi_{\mathcal{N}}(\mathrm{T})$.

The context-aware invariant waveform is given by [8], [9]

$$
\boldsymbol{\varphi}_{\mathrm{T}}=\frac{1}{\beta_{1}} \boldsymbol{P}_{\mathcal{N}, \mathrm{T}} \boldsymbol{e}_{1},
$$

where $\boldsymbol{P}_{\mathcal{N}, \mathrm{T}} \triangleq \boldsymbol{\Psi}_{\mathcal{N}}(\mathrm{T}) \boldsymbol{\Psi}_{\mathcal{N}}^{H}(\mathrm{~T})$ is the projector onto the noise subspace, $\boldsymbol{e}_{1}$ is a vector selecting the first column of $\boldsymbol{P}_{\mathcal{N}, \mathrm{T}}$, and $\beta_{1}$ is a scaling factor that guarantees unit norm. As introduced in [9], $\Psi_{\mathcal{N}}(\mathrm{T})$ has been adapted such that the main diagonal of $\boldsymbol{P}_{\mathcal{N}, \mathrm{T}}$ is sorted in decreasing order.

\section{B. Definition of Invariance}

It is worth noting that, following the reviewed methodology, opportunistic transmitting and receiving terminals might use different sensing bases, $\boldsymbol{\Psi}(\mathrm{T})$ and $\boldsymbol{\Psi}(\mathrm{R})$, respectively, and therefore both noise-subspace bases are also different. Even though, as proved in [8], [14], the reciprocal detection can be guaranteed thanks to the invariance property.

Let $\Psi_{\mathcal{N}}(\mathrm{T})$ and $\boldsymbol{\Psi}_{\mathcal{N}}(\mathrm{R})$ be bases of the noise subspaces at opportunistic transmitter and receiver denoted as $\mathcal{N}_{\mathrm{T}}$ and $\mathcal{N}_{\mathrm{R}}$, respectively. This two bases are obtained as previously 
introduced. Define the orthogonal projectors onto $\mathcal{N}_{\mathrm{T}}$ and $\mathcal{N}_{\mathrm{R}}$ as $\boldsymbol{P}_{\mathcal{N}, \mathrm{T}} \triangleq \boldsymbol{\Psi}_{\mathcal{N}}(\mathrm{T}) \boldsymbol{\Psi}_{\mathcal{N}}^{H}(\mathrm{~T})$ and $\boldsymbol{P}_{\mathcal{N}, \mathrm{R}} \triangleq \boldsymbol{\Psi}_{\mathcal{N}}(\mathrm{R}) \boldsymbol{\Psi}_{\mathcal{N}}^{H}(\mathrm{R})$. The chordal distance ${ }^{1}$ between $\mathcal{N}_{\mathrm{T}}$ and $\mathcal{N}_{\mathrm{R}}$ yields

$$
d_{\text {chordal }}\left(\mathcal{N}_{\mathrm{T}}, \mathcal{N}_{\mathrm{R}}\right)=\frac{1}{\sqrt{2}}\left\|\boldsymbol{P}_{\mathcal{N}, \mathrm{T}}-\boldsymbol{P}_{\mathcal{N}, \mathrm{R}}\right\|_{\mathrm{F}},
$$

with $\|\cdot\|_{\mathrm{F}}$ the Frobenius norm. Under the lack of sensing uncertainties [9], $\mathcal{N}_{\mathrm{T}}=\mathcal{N}_{\mathrm{R}}$ and, hence, $d_{\text {chordal }}\left(\mathcal{N}_{\mathrm{T}}, \mathcal{N}_{\mathrm{R}}\right)=0$. Necessarily, $\boldsymbol{P}_{\mathcal{N}, \mathrm{T}}=\boldsymbol{P}_{\mathcal{N}, \mathrm{R}}$ although bases $\boldsymbol{\Psi}(\mathrm{T})$ and $\boldsymbol{\Psi}(\mathrm{R})$ may be different. Thereby, any column of $\boldsymbol{P}_{\mathcal{N}, \mathrm{T}}$ and $\boldsymbol{P}_{\mathcal{N}, \mathrm{R}}$ are referred to as invariant waveforms. If $\mathcal{N}_{\mathrm{T}} \neq \mathcal{N}_{\mathrm{R}}$, opportunistic communication is only feasible when $\mathcal{N}_{\mathrm{T}} \cap \mathcal{N}_{\mathrm{R}} \neq \emptyset$. As reported in [8], [9], [14] and not considered in this work, the system suffers from subspace mismatching or uncertainties. Thus, the invariance only holds in the intersection of $\mathcal{N}_{\mathrm{T}}$ and $\mathcal{N}_{\mathrm{R}}$.

\section{Problem Formulation}

The invariance property previously defined is of paramount necessity to guarantee the detectability in uncoordinated and noncooperative opportunistic communication systems. Thus far, this invariance has only been proven in single-antenna scenarios [8] and in symmetric and uniform spatially-narrowband multi-antenna scenarios [11]. Since leveraging multi-antenna technology is of relevant importance to meet the demanding user-requirements in wireless networks, a natural question that arises is how antenna arrays geometry affects the invariance.

\section{A. System Model}

For simplicity of discussion, we consider a wideband multiple-input single-output (MISO) opportunistic communication scenario. We assume that: (i) opportunistic receiving terminal is located at the transmitter's far-field, and (ii) the size of temporal DoF ambient space $N$ is the same at both system ends. For the sake of generality, we consider that $L$-element transmitting antenna array has a 3D arbitrary geometry. Thus, even though the waveform design at receiving node can be cast as in [8], [9], we sketch herein the extension to multi-antenna terminals with arbitrary array geometries.

\section{B. Space-Time DoF Sensing and Waveform Design}

As first proved by the authors in [8], any orthonormal (random) basis of the whole ambient space is an efficient sensing matrix to appropriately detect the unused DoF under Gaussian conditions. Yet, in multichannel scenarios, the sensing matrix will have a particular structure due to the nature of the problem.

In order to generate a basis of the whole $N L$-dimensional space, we propose the following. Let $\boldsymbol{B} \in \mathbb{C}^{N \times N}$ be the frequency-bin matrix ${ }^{2}$ whose $\nu$-th column is given by

$$
\boldsymbol{b}_{\nu}=[\boldsymbol{B}]_{\nu} \triangleq\left[\begin{array}{lll}
1 e^{j \omega_{\nu}} & \cdots & e^{j(N-1) \omega_{\nu}}
\end{array}\right]^{T},
$$

\footnotetext{
${ }^{1}$ Let $\mathbb{G}(m, n)$ denote the (complex) Grassmann manifold of $n$-dimensional linear subspaces of the $m$-dimensional (complex) vector space. The chordal distance measures the extrinsic distance [15] between two subspaces belonging in $\mathbb{G}(m, n)$ and $\mathbb{G}\left(m, n^{\prime}\right)$, respectively. That is, the distance between two subspaces of the $m$-dimensional (complex) vector space. The chordal distance is induced by principal angles [16].

${ }^{2}$ To avoid the effects of frequency-selective channels, the matrix $\boldsymbol{B}$ can be particularized to work with OFDM modulations with cyclic prefix.
}

and let $\boldsymbol{S}_{\nu}(\boldsymbol{\theta}, \boldsymbol{\phi}) \in \mathbb{C}^{L \times L}$ be the array steering matrix at frequency $\omega_{\nu}$, where the $\ell$-th column is given by

$$
\left[\boldsymbol{S}_{\nu}(\boldsymbol{\theta}, \boldsymbol{\phi})\right]_{\ell}=\boldsymbol{a}_{\nu}\left(\theta_{\ell}, \phi_{\ell}\right) \triangleq\left[\begin{array}{lll}
1 e^{j \boldsymbol{k}_{\nu, \ell}^{T} \boldsymbol{r}_{1}} & \cdots & e^{j \boldsymbol{k}_{\nu, \ell}^{T} \boldsymbol{r}_{L-1}}
\end{array}\right]^{T},
$$

where $\boldsymbol{r}_{\ell} \triangleq\left(x_{\ell}, y_{\ell}, z_{\ell}\right)$ are the coordinates of the $\ell$-th sensor and $\boldsymbol{k}_{\nu, \ell}$ is the wavenumber vector corresponding to the $\ell$-th dimension of the spatial ambient space at frequency $\omega_{\nu}$. Letting $\lambda_{\nu}$ the wavelength associated to $\omega_{\nu}, \boldsymbol{k}_{\nu, \ell}$ is defined as

$$
\boldsymbol{k}_{\nu, \ell} \triangleq \frac{2 \pi}{\lambda_{\nu}}\left[\sin \left(\theta_{\ell}\right) \cos \left(\phi_{\ell}\right), \sin \left(\theta_{\ell}\right) \sin \left(\phi_{\ell}\right), \cos \left(\theta_{\ell}\right)\right]^{T} .
$$

It is worth noting that, to generate the whole $L$-dimensional spatial ambient space at each frequency, $\boldsymbol{S}_{\nu}(\boldsymbol{\theta}, \boldsymbol{\phi})$ has to be a full-rank matrix. That is, we need to find the set of $\ell=1, \ldots, L$ direction pairs $\left(\theta_{\ell}, \phi_{\ell}\right)$ that meet the full-rank condition. These directions are then collected in vectors $\boldsymbol{\theta}$ and $\phi$, respectively. Finally, to take the temporal dimension into account, we define the multi-frequency steering matrix ${ }^{3}$ as

$$
\boldsymbol{S}(\boldsymbol{\theta}, \boldsymbol{\phi} ; \boldsymbol{\omega}) \triangleq\left[\boldsymbol{b}_{0} \otimes \boldsymbol{S}_{0}(\boldsymbol{\theta}, \boldsymbol{\phi}) \cdots \boldsymbol{b}_{N-1} \otimes \boldsymbol{S}_{N-1}(\boldsymbol{\theta}, \boldsymbol{\phi})\right] .
$$

Even though a basis of the whole $N L$-dimensional space can be constructed by means of the presented procedure, it is worth noticing that (6) is not orthonormal. Thus, it is not an efficient matrix for detecting the available DoF nor for designing the total least-squares waveform, robust to sensing uncertainties.

Leveraging a canonical transformation [17], an orthonormal basis of the whole $N L$-dimensional space can be found as

$$
\boldsymbol{\Psi}=\boldsymbol{T} \boldsymbol{S}(\boldsymbol{\theta}, \boldsymbol{\phi} ; \boldsymbol{\omega}) \in \mathbb{C}^{N L \times N L} .
$$

As concluded in [17], canonical coordinates constitute a complete set of invariants for the matrix $\boldsymbol{S}(\boldsymbol{\theta}, \boldsymbol{\phi} ; \boldsymbol{\omega}) \boldsymbol{S}^{H}(\boldsymbol{\theta}, \boldsymbol{\phi} ; \boldsymbol{\omega})$ under the group of full-rank transformations. The latter is of paramount importance for our purpose. Since the orthonormal basis $\Psi$ presents the aforementioned invariances, opportunistic multi-antenna terminals might leverage $\Psi$ to uniquely sense the whole $N L$-dimensional space. However, the sensing performance will truly rely on the chosen sensing mechanism [18], [19]. We would like to remark that the design of space-time sensing bases is beyond the scope of this work and it is only sketched herein for notation purposes.

Once basis $\Psi$ has been generated, a multi-antenna terminal (herein, the transmitter) can unambiguously divide (7) into

$$
\boldsymbol{\Psi}(\mathrm{T})=\left[\begin{array}{l:l}
\boldsymbol{\Psi}_{\mathcal{S}}(\mathrm{T}) & \left.\boldsymbol{\Psi}_{\mathcal{N}}(\mathrm{T})\right]
\end{array}\right]
$$

It is noteworthy that the non-ambiguous DoF sensing is possible thanks to the use of the canonical basis and its invariance property. However, sensing imperfections due to monitoring conditions and the robustness of these mechanisms cannot be overcome by the use of the canonical basis. Recalling Sec. I-A, the space-time signaling pattern is obtained as

$$
\boldsymbol{\varphi}_{\mathrm{T}}=\frac{1}{\beta_{1}} \boldsymbol{P}_{\mathcal{N}, \mathrm{T}} \boldsymbol{e}_{1},
$$

with $\boldsymbol{\varphi}_{\mathrm{T}} \in \mathbb{C}^{N L}$ and $\boldsymbol{P}_{\mathcal{N}, \mathrm{T}}=\boldsymbol{\Psi}_{\mathcal{N}}(\mathrm{T}) \boldsymbol{\Psi}_{\mathcal{N}}^{H}(\mathrm{~T})$ is the projector

\footnotetext{
${ }^{3}$ The construction of this matrix requires knowing the array geometry. Actually, since observations' autocorrelation eigenmatrix can be used as an orthonormal basis, the knowledge on array geometry is not strictly needed. Yet, we assume this knowledge herein to analyze its impact.
} 


$$
\boldsymbol{S}(\boldsymbol{\theta}, \boldsymbol{\phi} ; \boldsymbol{\omega})=\left[\begin{array}{cccc}
\boldsymbol{S}_{0}(\boldsymbol{\theta}, \boldsymbol{\phi}) & \boldsymbol{S}_{1}(\boldsymbol{\theta}, \boldsymbol{\phi}) & \cdots & \boldsymbol{S}_{N-1}(\boldsymbol{\theta}, \boldsymbol{\phi}) \\
\boldsymbol{S}_{0}(\boldsymbol{\theta}, \boldsymbol{\phi}) & \boldsymbol{S}_{1}(\boldsymbol{\theta}, \boldsymbol{\phi}) & \cdots & \boldsymbol{S}_{N-1}(\boldsymbol{\theta}, \boldsymbol{\phi}) \\
\vdots & \vdots & \cdots & \vdots \\
\boldsymbol{S}_{0}(\boldsymbol{\theta}, \boldsymbol{\phi}) & \boldsymbol{S}_{1}(\boldsymbol{\theta}, \boldsymbol{\phi}) & \cdots & \boldsymbol{S}_{N-1}(\boldsymbol{\theta}, \boldsymbol{\phi})
\end{array}\right] \odot\left[\begin{array}{cccc}
\mathbf{1}_{L L}\left[\boldsymbol{b}_{0}\right]_{1} & \mathbf{1}_{L L}\left[\boldsymbol{b}_{1}\right]_{1} & \cdots & \mathbf{1}_{L L}\left[\boldsymbol{b}_{N-1}\right]_{1} \\
\mathbf{1}_{L L}\left[\boldsymbol{b}_{0}\right]_{2} & \mathbf{1}_{L L}\left[\boldsymbol{b}_{1}\right]_{2} & \cdots & \mathbf{1}_{L L}\left[\boldsymbol{b}_{N-1}\right]_{2} \\
\vdots & \vdots & \cdots & \vdots \\
\mathbf{1}_{L L}\left[\boldsymbol{b}_{0}\right]_{N} & \mathbf{1}_{L L}\left[\boldsymbol{b}_{1}\right]_{N} & \cdots & \mathbf{1}_{L L}\left[\boldsymbol{b}_{N-1}\right]_{N}
\end{array}\right]
$$

onto the space-time noise subspace sensed at opportunistic transmitter. Notice that (9) robustly exploits the available DoF correctly sensed as available whereas minimizes the impact on those incorrectly detected as available DoF (cf. [8], [9]). To analyze the impact of transmitting array geometry at a single-antenna far-field opportunistic node, we consider the transmission of (9) through a wideband MISO channel.

\section{IMPACT OF the ANTENNA ARRAy GEOMETRY}

We assume that opportunistic receiving terminal is located at $\left(\theta_{\mathrm{R}}, \phi_{\mathrm{R}}\right)$ with respect to opportunistic transmitter's broadside. We define the time-domain MISO response as

$$
\boldsymbol{h}[n] \triangleq \frac{1}{N \sqrt{L}} \sum_{\nu=0}^{N-1} \boldsymbol{a}_{\nu}\left(\theta_{\mathrm{R}}, \phi_{\mathrm{R}}\right) e^{j 2 \pi \nu \frac{n}{N}},
$$

where $\nu$ indexes the frequency and $\boldsymbol{a}_{\nu}\left(\theta_{\mathrm{R}}, \phi_{\mathrm{R}}\right)$ is the steering vector pointing the direction $\left(\theta_{\mathrm{R}}, \phi_{\mathrm{R}}\right)$. For $n=0, \ldots, N-1$, the MISO response matrix is defined as

$$
\boldsymbol{H} \triangleq\left[\begin{array}{ccc}
\boldsymbol{h}[0] & & \mathbf{0} \\
& \ddots & \\
\mathbf{0} & & \boldsymbol{h}[N-1]
\end{array}\right] \in \mathbb{C}^{N L \times N} .
$$

Thus, the time-domain received signal is given by

$$
\boldsymbol{y}=\boldsymbol{H}^{H} \boldsymbol{\varphi}_{\mathrm{T}}+\boldsymbol{v} \in \mathbb{C}^{N},
$$

where $\boldsymbol{v}$ is the noise-plus-interference term. Notice that array geometry is embedded in both $\boldsymbol{H}$ and $\varphi_{\mathrm{T}}$. However, as detailed in Sec. II-B, the impact of array geometry on $\varphi_{\mathrm{T}}$ is hidden in the orthonormal noise-subspace basis $\Psi_{\mathcal{N}}(\mathrm{T})$.

By carefully analyzing the construction of matrix $S(\theta, \phi ; \omega)$ in (6), we note that it can be written as in (13), on the top of this page. Notice that $\mathbf{1}_{k k}$ is the $k \times k$ all-ones matrix, $\left[\boldsymbol{b}_{\nu}\right]_{n}$ is the $n$-th entry of vector $\boldsymbol{b}_{\nu}$ (3), and $\odot$ is the Schur-Hadamard (element-wise) product.

The latter is of paramount importance. Since the steering matrix (4) is replicated $N$ times, $\boldsymbol{S}(\boldsymbol{\theta}, \boldsymbol{\phi} ; \boldsymbol{\omega})$ will be structured in $N$ blocks of $L$ rows, where each block only differs from the previous one by a phase term. Therefore, it can be proven that the $n$-th element of (12) can be written as

$$
y[n]=\frac{1}{N \sqrt{L}} \sum_{\nu=0}^{N-1} \boldsymbol{a}_{\nu}^{H}\left(\theta_{\mathrm{R}}, \phi_{\mathrm{R}}\right) \boldsymbol{S}_{\nu}(\boldsymbol{\theta}, \boldsymbol{\phi}) \boldsymbol{\lambda}_{\nu},
$$

where $\boldsymbol{\lambda}_{\nu}$ is suitably combining the elements of $\boldsymbol{S}_{\nu}(\boldsymbol{\theta}, \boldsymbol{\phi})$ for those couples $\left(\left(\theta_{\ell}, \phi_{\ell}\right) ; \omega_{\nu}\right)$ sensed as available, i.e.

$$
\boldsymbol{\lambda}_{\nu}=\left\{\begin{array}{cl}
\mathbf{0}, & \text { if }\left(\left(\theta_{\ell}, \phi_{\ell}\right) ; \omega_{\nu}\right) \text { is occupied } \\
\gamma_{\nu} \boldsymbol{S}_{\nu}^{+}(\boldsymbol{\theta}, \boldsymbol{\phi}) \boldsymbol{e}_{1}, & \text { otherwise }
\end{array}\right.
$$

with $\boldsymbol{A}^{+}$the Moore-Penrose pseudo-inverse of matrix $\boldsymbol{A}$. The formal proof of (14) is omitted for space reasons, whereas the derivation of (15) is sketched in Appendix. At this point, we can express (12) in terms of steering vectors.

To analyze the impact of array geometry on (12), or (14), we would like to separate the steering vector into a physical array-only dependent term and a spatial DoF-only dependent term, which is possible thanks to array manifold separation.

\section{A. Array Manifold Separation}

The steering vector of a non-polarized ${ }^{4} L$-element antenna array is, in general, given by [23]

$$
\boldsymbol{a}_{\nu}(\theta, \phi)=\frac{1}{\sqrt{L}}\left[e^{j \boldsymbol{k}_{\nu}^{T} \boldsymbol{r}_{0}} \cdots e^{j \boldsymbol{k}_{\nu}^{T} \boldsymbol{r}_{\ell}} \cdots e^{j \boldsymbol{k}_{\nu}^{T} \boldsymbol{r}_{L-1}}\right]^{T}
$$

where $\boldsymbol{k}_{\nu}$ is the wavenumber vector at frequency $\omega_{\nu}$ and $\boldsymbol{r}_{\ell}$ is the position vector of the $\ell$-th array element with respect to the phase reference element (typically $\boldsymbol{r}_{0}$ ). Notice that (16) depends on both array geometry and directions $(\theta, \phi)$. Thus, the so-called array manifold at frequency $\omega_{\nu}$,

$$
\mathcal{A}\left(\omega_{\nu}\right) \triangleq\left\{\boldsymbol{a}_{\nu}(\theta, \phi):(\theta, \phi) \in[-\pi, \pi]\right\},
$$

is also geometry-dependent. As introduced in [24], the array signal model can be generalized by means of the so-called wavefield model. Therefore, (16) admits the decomposition

$$
\boldsymbol{a}_{\nu}(\theta, \phi)=\boldsymbol{G}_{\nu} \boldsymbol{d}(\theta, \phi),
$$

where the sampling matrix $\boldsymbol{G}_{\nu}$ describes the physical antenna array, whereas the coefficient vector $\boldsymbol{d}(\theta, \phi)$ uniquely describes the wavefield at direction $(\theta, \phi) \cdot \boldsymbol{G}_{\nu}$ and $\boldsymbol{d}(\theta, \phi)$ are independent. According to the literature, (18) is a Fourier series expansion of the array manifold. Thus, $\boldsymbol{d}(\theta, \phi) \in \mathcal{H}$ is a function on a Hilbert space, and $\boldsymbol{G}_{\nu}: \mathcal{H} \rightarrow \mathbb{C}^{L}$ is nothing but the mapping of functions defined on $\mathcal{H}$ onto the $L$-dimensional complex space. In the sequel, we leverage (18) to rewrite (14) and be able to study the impact of antenna array geometry.

\section{B. Analysis of the Array-Geometry Invariance}

The objective of this work is to prove that time-domain received signal (12) does not depend on transmitting array geometry. Equivalently, we can prove that each of the $n=$ $0, \ldots, N-1$ entries is independent of the array geometry. Hence, focusing on (14), we may write it as

\footnotetext{
${ }^{4}$ Throughout this work, we focus the whole discussion on non-polarized antenna arrays. Notice that, when polarization is taken into consideration, one may observe a larger array manifold, since array steering vectors also depend on the polarization angles. In communications, array polarization translates into doubling the spatial DoF [20]. For further details on polarized-array manifold separation, the reader is referred to [21], [22].
} 


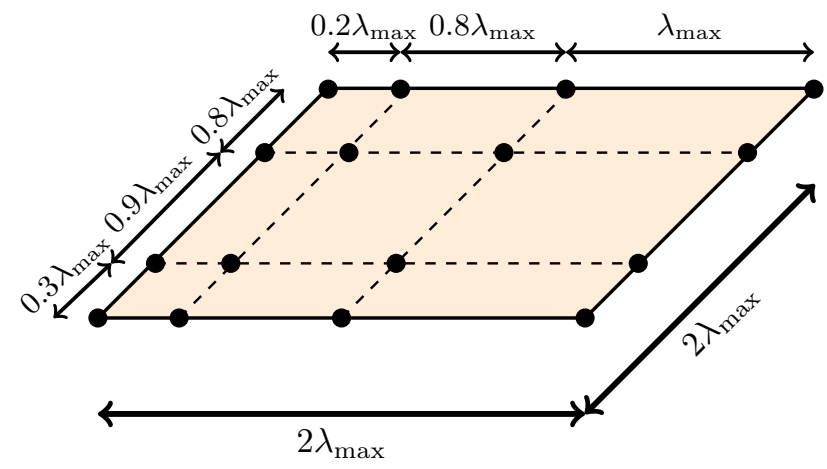

Fig. 1: $4 \times 4$ non-uniform grid considered in this work to generate opportunistic transmitting arrays. At each simulation, 4 elements are randomly chosen.

$$
y[n]=\frac{1}{N \sqrt{L}} \sum_{\nu=0}^{N-1} \boldsymbol{p}_{\nu}^{H} \boldsymbol{\lambda}_{\nu},
$$

where $\boldsymbol{\lambda}_{\nu}$ is defiend in (15), and

$$
\boldsymbol{p}_{\nu} \triangleq \boldsymbol{S}_{\nu}^{H}(\boldsymbol{\theta}, \boldsymbol{\phi}) \boldsymbol{a}_{\nu}\left(\theta_{\mathrm{R}}, \phi_{\mathrm{R}}\right)
$$

is the sample correlation vector between all direction in $(\boldsymbol{\theta}, \boldsymbol{\phi})$ and the reference $\left(\theta_{\mathrm{R}}, \phi_{\mathrm{R}}\right)$. Leveraging array manifold separation (18), we have that

$$
\begin{aligned}
\boldsymbol{a}_{\nu}\left(\theta_{\mathrm{R}}, \phi_{\mathrm{R}}\right) & =\boldsymbol{G}_{\nu} \boldsymbol{d}\left(\theta_{\mathrm{R}}, \phi_{\mathrm{R}}\right), \\
\boldsymbol{S}_{\nu}(\boldsymbol{\theta}, \boldsymbol{\phi}) & =\boldsymbol{G}_{\nu} \boldsymbol{D}(\boldsymbol{\theta}, \boldsymbol{\phi}),
\end{aligned}
$$

where $\boldsymbol{D}(\boldsymbol{\theta}, \boldsymbol{\phi})$ contains $L$ coefficient vectors, i.e.

$$
\boldsymbol{D}(\boldsymbol{\theta}, \boldsymbol{\phi}) \triangleq\left[\boldsymbol{d}\left(\theta_{1}, \phi_{1}\right) \cdots \boldsymbol{d}\left(\theta_{L}, \phi_{L}\right)\right] .
$$

Recall that array geometry is encompassed in $\boldsymbol{G}_{\nu}$, whereas $\boldsymbol{d}(\theta, \phi)$ only depends on spatial DoF. Since $\boldsymbol{G}_{\nu}$ constitutes an orthonormal expansion, vector $\boldsymbol{p}_{\nu}$ in (20) yields

$$
\begin{aligned}
\boldsymbol{p}_{\nu} & =\boldsymbol{D}^{H}(\boldsymbol{\theta}, \boldsymbol{\phi}) \boldsymbol{G}_{\nu}^{H} \boldsymbol{G}_{\nu} \boldsymbol{d}\left(\theta_{\mathrm{R}}, \phi_{\mathrm{R}}\right) \\
& =\boldsymbol{D}^{H}(\boldsymbol{\theta}, \boldsymbol{\phi}) \boldsymbol{d}\left(\theta_{\mathrm{R}}, \phi_{\mathrm{R}}\right),
\end{aligned}
$$

where (25) follows from the orthonormality of expansion basis $\boldsymbol{G}_{\nu}$. Therefore, we may conclude that $\boldsymbol{p}_{\nu}$ does not rely on array geometry. Nevertheless, we need to study if $\boldsymbol{\lambda}_{\nu}$ is (in)dependent of array geometry. For that purpose, recalling (15), we only need to study the Moore-Penrose pseudo-inverse of $\boldsymbol{S}_{\nu}(\boldsymbol{\theta}, \boldsymbol{\phi})$.

Recall that $\boldsymbol{S}_{\nu}^{+}(\boldsymbol{\theta}, \boldsymbol{\phi})=\left(\boldsymbol{S}_{\nu}^{H}(\boldsymbol{\theta}, \boldsymbol{\phi}) \boldsymbol{S}_{\nu}(\boldsymbol{\theta}, \boldsymbol{\phi})\right)^{-1} \boldsymbol{S}_{\nu}^{H}(\boldsymbol{\theta}, \boldsymbol{\phi})$. We first study the term $\left(\boldsymbol{S}_{\nu}^{H}(\boldsymbol{\theta}, \boldsymbol{\phi}) \boldsymbol{S}_{\nu}(\boldsymbol{\theta}, \boldsymbol{\phi})\right)^{-1}$. Using (22), this term can be written as

$$
\left(\boldsymbol{D}^{H}(\boldsymbol{\theta}, \boldsymbol{\phi}) \boldsymbol{G}_{\nu}^{H} \boldsymbol{G}_{\nu} \boldsymbol{D}(\boldsymbol{\theta}, \boldsymbol{\phi})\right)^{-1} .
$$

Taking into account the orthonormality of $\boldsymbol{G}_{\nu}$, (26) yields

$$
\left(\boldsymbol{D}^{H}(\boldsymbol{\theta}, \boldsymbol{\phi}) \boldsymbol{D}(\boldsymbol{\theta}, \boldsymbol{\phi})\right)^{-1}
$$

which is independent of array geometry. Hence, the possible dependence on array geometry can only be found in $\boldsymbol{S}_{\nu}^{H}(\boldsymbol{\theta}, \boldsymbol{\phi})$. Recalling (15), and using (22) and (27), we have that

$$
\boldsymbol{\lambda}_{\nu}=\gamma_{\nu}\left(\boldsymbol{D}^{H}(\boldsymbol{\theta}, \boldsymbol{\phi}) \boldsymbol{D}(\boldsymbol{\theta}, \boldsymbol{\phi})\right)^{-1} \boldsymbol{D}^{H}(\boldsymbol{\theta}, \boldsymbol{\phi}) \boldsymbol{G}_{\nu}^{H} \boldsymbol{e}_{1} .
$$

Since the term $\boldsymbol{D}^{H}(\boldsymbol{\theta}, \phi)$ only depends on spatial DoF, we have to study the impact of $G_{\nu}^{H} e_{1}$. Since vector $\boldsymbol{e}_{1}$ selects the

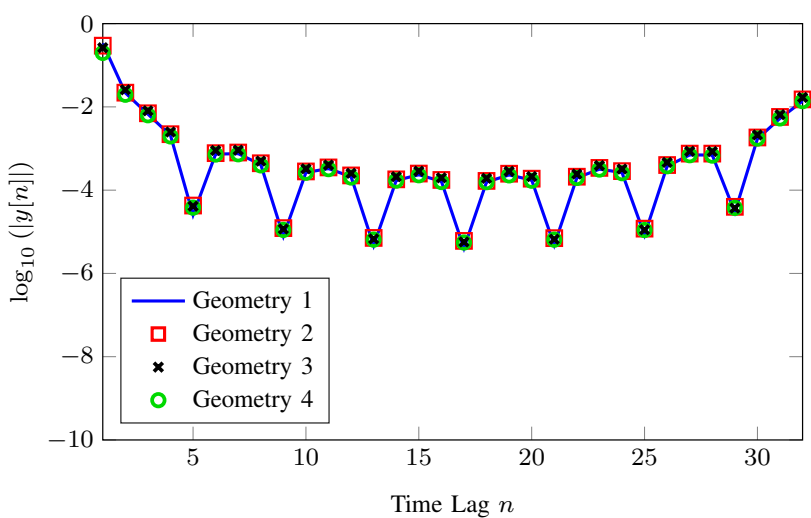

Fig. 2: Magnitude of opportunistic received signal $y[n]$ (14) at different time lags and for different transmitting array geometries. Notice that $N=32$.

first column of the multiplying matrix (in this case, $\boldsymbol{G}_{\nu}^{H}$ ), the term $\boldsymbol{G}_{\nu}^{H} \boldsymbol{e}_{1}$ is just the first row of sampling matrix $\boldsymbol{G}_{\nu}$.

By carefully reviewing the literature on array manifold separation theory, we realize that rows of $\boldsymbol{G}_{\nu}$ describe the array architecture at a given mode (both its geometry and nonidealities, such as calibration errors). The columns of $\boldsymbol{G}_{\nu}$ are related to the excitation modes that perfectly map a function defined on $\mathcal{H}$ onto $\mathbb{C}^{L}$. The number of significant modes is limited and known as spatial DoF [20]. Hence, each row of $\boldsymbol{G}_{\nu}$ represents the spatial DoF at a given sensor, whereas its columns describe how spatial DoF are geometrically organized.

Thus, taking into consideration (28), since $\boldsymbol{\lambda}_{\nu}$ depends on the first row of $\boldsymbol{G}_{\nu}$, we may conclude that $y[n]$ in (14) is invariant to transmitting array geometry. Specifically, the time-domain received signal can be seen as a coherent combination of the spatial DoF observed from the array element associated to the first row of $\boldsymbol{G}_{\nu}$ (typically, the phase reference element). Since time-domain invariance is preserved, this result constitutes a generalization of the concept of invariance.

\section{Simulation Analysis}

This section is devoted to numerically illustrating the arraygeometry invariance proven in this paper. For this purpose, we consider that opportunistic terminal is equipped with a 2D arbitrary antenna array of 4 isotropic elements. These 4 elements have been randomly chosen from the non-uniform $4 \times 4$ grid depicted in Fig. 1. It is worth noting that $\lambda_{\max }$ refers to the wavelength at the highest frequency considered.

In order to illustrate the theoretical statement, we design 4 different space-time signaling patterns, each of them with a different array geometry. Nevertheless, we fix the size of the time-domain ambient space to $N=32 \mathrm{DoF}$. Thus, the size of the space-time ambient space is $N L=128 \mathrm{DoF}$.

First, once the 4 elements have been chosen, we construct the sensing basis according to the methodology sketched in Sec. II-B. In order to guarantee that the spatial DoF sample correlation, observed in (25) and (27) through inner products $\boldsymbol{D}^{H}(\boldsymbol{\theta}, \boldsymbol{\phi}) \boldsymbol{d}\left(\theta_{\mathrm{R}}, \phi_{\mathrm{R}}\right)$ and $\boldsymbol{D}^{H}(\boldsymbol{\theta}, \boldsymbol{\phi}) \boldsymbol{D}(\boldsymbol{\theta}, \boldsymbol{\phi})$, respectively, is the same in each case, we select the same set of $L$ azimuthelevation pairs $\left(\theta_{\ell}, \phi_{\ell}\right)$, collected in vectors $(\boldsymbol{\theta}, \boldsymbol{\phi})$. 
Then, we randomly discard the $25 \%$ of space-time DoF, as a result of the sensing mechanism. We also discard the same DoF in each simulation. The analysis of sensing is beyond the scope of this work. Therefore, we do not care about how it has been performed, but only what is the noise subspace.

Leveraging the noise-subspace orthonormal basis, which contains the $75 \%$ of the total space-time ambient space, we design the signaling pattern according to (9). Then, we transmit this waveform through the MISO opportunistic channel, modeled in (11), assuming $\theta_{\mathrm{R}}=30^{\circ}$ and $\phi_{\mathrm{R}}=60^{\circ}$. In order to purely observe the effects of array geometry, we work in (very) high signal-to-interference-plus-noise ratio regimes.

In Fig. 2, we depict the magnitude of received signal $y[n]$. We observe that the plots are extremely similar regardless the considered array geometry. Yet, very small differences can be appreciated. The causes of this differences are numerical stability (specially in those scenarios with $\left(d / \lambda_{\max }\right)>1 / 2$ ), and the possible grating lobes at some frequencies. When the latter are notable, larger differences are observed, even though the shape of the received signal's magnitude is preserved.

\section{Conclusions}

In this paper, we have demonstrated that time-domain invariance of total least-squares space-time signaling patterns is preserved regardless the antenna array geometry. The analysis has been addressed in an asymmetric wideband multichannel communication scenario (MISO) and exploiting the theory of array manifold separation. As a result, we have observed that time-domain received signal is a linear combination of information spread among spatial DoF, regardless how the latter are geometrically structured.

\section{APPENDIX}

Notice that $\boldsymbol{\lambda}_{\nu}=\mathbf{0}$ for all those couples $\left(\left(\theta_{\ell}, \phi_{\ell}\right) ; \omega_{\nu}\right)$ sensed as occupied. This follows from the sensing previously performed. Hence, we only need to design the solution for the available couples $\left(\left(\theta_{\ell}, \phi_{\ell}\right) ; \omega_{\nu}\right)$, i.e. the noise subspace.

Let $\boldsymbol{S}_{\mathcal{N}}(\boldsymbol{\theta}, \boldsymbol{\phi} ; \boldsymbol{\omega})=\boldsymbol{S}\left((\boldsymbol{\theta}, \boldsymbol{\phi})_{\mathcal{N}} ; \boldsymbol{\omega}_{\mathcal{N}}\right)$, where $\left((\boldsymbol{\theta}, \boldsymbol{\phi})_{\mathcal{N}} ; \boldsymbol{\omega}_{\mathcal{N}}\right)$ represents all available couples $\left(\left(\theta_{\ell}, \phi_{\ell}\right) ; \omega_{\nu}\right)$.

As in [8], [10], [11], the opportunistic signaling pattern is designed as $\boldsymbol{\varphi}=\boldsymbol{S}_{\mathcal{N}}(\boldsymbol{\theta}, \boldsymbol{\phi} ; \boldsymbol{\omega}) \boldsymbol{\lambda}$. Mathematically, this problem can be formulated as [10]

$$
\boldsymbol{\varphi}=\underset{\boldsymbol{\lambda}}{\arg \min }\left\|\boldsymbol{S}_{\mathcal{N}}(\boldsymbol{\theta}, \boldsymbol{\phi} ; \boldsymbol{\omega}) \boldsymbol{\lambda}\right\|^{2} \text { subject to } \boldsymbol{\varphi}^{H} \boldsymbol{e}_{1}=1
$$

where the last term is a non-trivial design constraint. The solution, which follows from minimizing the Lagrangian associated to (29) [10], is given by

$$
\boldsymbol{\lambda}=\mu \boldsymbol{S}_{\mathcal{N}}^{+}(\boldsymbol{\theta}, \boldsymbol{\phi} ; \boldsymbol{\omega}) \boldsymbol{e}_{1},
$$

with $\mu$ a scaling factor. By developing (30), and accounting for the structure of steering matrix (6), we find that $\boldsymbol{\lambda}$ is structured in as blocks as length of $\boldsymbol{\omega}_{\nu}$. Let $\boldsymbol{\lambda}_{\nu}$ be one of these blocks. It is straightforward to see that

$$
\boldsymbol{\lambda}_{\nu}=\gamma_{\nu} \boldsymbol{S}_{\nu}^{+}\left((\boldsymbol{\theta}, \boldsymbol{\phi})_{\mathcal{N}}\right) \boldsymbol{e}_{1}
$$

where $e_{1}=[1,0 \ldots, 0]^{T}$, of appropriate dimensions, selects the first column of $\boldsymbol{S}_{\nu}^{+}\left((\boldsymbol{\theta}, \boldsymbol{\phi})_{\mathcal{N}}\right)$ and $\gamma_{\nu}[n] \triangleq\left(\left\|\boldsymbol{b}_{v}\right\|^{2}\right)^{-1}\left[\boldsymbol{b}_{v}\right]_{1}^{*}$.

\section{REFERENCES}

[1] S. Goguri, D. Ogbe, S. Dasgupta, R. Mudumbai, D. R. Brown, D. J. Love, and U. Madhow, "Optimal precoder design for distributed transmit beamforming over frequency-selective channels," IEEE Trans. Wireless Commun., vol. 17, no. 11, pp. 7759-7773, Nov 2018.

[2] Y. Wang, Y. . P. Hong, and W. Chen, "Dynamic transmission policy for multi-pair cooperative device-to-device communication with blockdiagonalization precoding," IEEE Trans. Wireless Commun., vol. 18, no. 6, pp. 3034-3048, June 2019.

[3] I. Wang and D. N. C. Tse, "Interference mitigation through limited transmitter cooperation," IEEE Trans. Inf. Theory, vol. 57, no. 5, pp. 2941-2965, May 2011.

[4] S. A. Ayoughi, W. Yu, S. R. Khosravirad, and H. Viswanathan, "Interference mitigation for ultrareliable low-latency wireless communication," IEEE J. Sel. Areas Commun., vol. 37, no. 4, pp. 869-880, April 2019.

[5] D. Zahavi and R. Dabora, "On cooperation and interference in the weak interference regime," IEEE Trans. Inf. Theory, vol. 63, no. 6, pp. 38943922, June 2017.

[6] J. Park, N. Lee, and R. W. Heath, "Feedback design for multi-antenna $k$-tier heterogeneous downlink cellular networks," IEEE Trans. Wireless Commun., vol. 17, no. 6, pp. 3861-3876, June 2018.

[7] A. Lozano, R. W. Heath, and J. G. Andrews, "Fundamental limits of cooperation," IEEE Trans. Inf. Theory, vol. 59, no. 9, pp. 5213-5226, Sep. 2013.

[8] J. Borras and G. Vazquez, "Decentralized shaping for pilot generation and detection in opportunistic communications," in 2019 IEEE Int. Conf. Commun. (ICC), May 2019, pp. 1-5.

[9] J. Borras and G. Vazquez, "Interference mitigation under degrees-offreedom sensing uncertainties in opportunistic transmission," in 2019 IEEE Global Commun. Conf. (GLOBECOM), 2019, pp. 1-5.

[10] J. Borras, J. Font-Segura, J. Riba, and G. Vazquez, "Dimension spreading for coherent opportunistic communications," in 2017 51st Asilomar Conf. Signals, Syst. Comput., Oct 2017, pp. 1940-1944.

[11] J. Borras and G. Vazquez, "Uncoordinated space-frequency pilot design for multi-antenna wideband opportunistic communications," in 2018 IEEE 19th Int. Workshop Signal Process. Adv. Wireless Commun. (SPAWC), Jun 2018, pp. 1-5.

[12] R. G. Gallager, Information Theory and Reliable Communications. John Wiley \& Sons, 1968.

[13] D. Tse and P. Viswanath, Fundamentals of Wireless Communication. Cambridge University Press, 2005.

[14] J. Borras and G. Vazquez, "Distributed feedback-aided subspace concurrent opportunistic communications," in 2019 IEEE 20th Int. Workshop Signal Process. Adv. Wireless Commun. (SPAWC), Jul 2019, pp. 1-5.

[15] A. Srivastava and E. Klassen, "Monte Carlo extrinsic estimators of manifold-valued parameters," IEEE Trans. Signal Process., vol. 50, no. 2, pp. 299-308, Feb 2002.

[16] G. H. Golub and C. F. V. Loan, Matrix Computations. The Johns Hopkins University Press, 1996.

[17] L. L. Scharf and C. T. Mullis, "Canonical coordinates and the geometry of inference, rate, and capacity," IEEE Trans. Signal Process., vol. 48, no. 3, pp. 824-831, March 2000.

[18] A. Tajer, R. M. Castro, and X. Wang, "Adaptive sensing of congested spectrum bands," IEEE Trans. Inf. Theory, vol. 58, no. 9, Sep. 2012.

[19] J. Font-Segura, G. Vázquez, and J. Riba, "Single and multi-frequency wideband spectrum sensing with side-information," IET Signal Process., vol. 8, no. 8, pp. 831-843, 2014.

[20] A. S. Y. Poon, R. W. Brodersen, and D. N. C. Tse, "Degrees of freedom in multiple-antenna channels: a signal space approach," IEEE Trans. Inf. Theory, vol. 51, no. 2, pp. 523-536, Feb 2005.

[21] M. Costa, A. Richter, and V. Koivunen, "DoA and polarization estimation for arbitrary array configurations," IEEE Trans. Signal Process., vol. 60, no. 5, pp. 2330-2343, May 2012.

[22] B. Friedlander, "Antenna array manifolds for high-resolution direction finding," IEEE Trans. Signal Process., vol. 66, no. 4, pp. 923-932, Feb 2018.

[23] H. L. Van Trees, Optimum array processing: Part IV of detection, estimation, and modulation theory. John Wiley \& Sons, 2002.

[24] M. A. Doron and E. Doron, "Wavefield modeling and array processing .i. spatial sampling," IEEE Trans. Signal Process., vol. 42, no. 10, pp. 2549-2559, Oct 1994. 